\title{
Network Pharmacology and Bioinformatics Methods Reveal the Mechanism of Zao-Jiao-Ci in the Treatment of LSCC
}

\author{
Feng Xiang $\left(\mathbb{D},{ }^{1}\right.$ Linman Li $\mathbb{D}{ }^{2}{ }^{2}$ Jieling Lin $\left(\mathbb{D},{ }^{2}\right.$ Shasha Li $\mathbb{D}^{2},{ }^{2}$ and Guiyuan Peng $\mathbb{D}^{3}$ \\ ${ }^{1}$ Department of Otolaryngology, YangZhou Hospital of Traditional Chinese Medicine, JiangSu, YangZhou, China \\ ${ }^{2}$ The Second Clinical College, Guangzhou University of Chinese Medicine, Guangzhou, Guangdong, China \\ ${ }^{3}$ Department of Otolaryngology, Guangdong Province Hospital of Chinese Medicine, Guangzhou, Guangdong, China \\ Correspondence should be addressed to Shasha Li; liss326818@gzucm.edu.cn and Guiyuan Peng; zypgy@126.com
}

Received 10 September 2020; Accepted 8 June 2021; Published 28 June 2021

Academic Editor: Dan Zhao

Copyright (c) 2021 Feng Xiang et al. This is an open access article distributed under the Creative Commons Attribution License, which permits unrestricted use, distribution, and reproduction in any medium, provided the original work is properly cited.

Objective. Zao-Jiao-Ci (ZJC), a traditional Chinese medicine, is considered as a promising candidate to treat laryngeal squamous cell carcinoma (LSCC). However, the underlying molecular mechanism remains unclear. Methods. Gene expression profiles of GSE36668 were available from the GEO database, and differentially expressed genes (DEGs) of LSCC were obtained by $R$ package; subsequently, enrichment analysis on KEGG and GO of DEGs was performed. The active ingredients of ZJC were screened from the TCMSP database, and the matched candidate targets were obtained by PharmMapper. Furthermore, we constructed proteinprotein interaction (PPI) networks of DEGs and candidate targets, respectively, and we screened the core network from the merged network through combining the two PPI networks using Cytoscape 3.7.2. The key targets derived from the core network were analyzed to find out the associated KEGG signal enrichment pathway. By the GEPIA online website, Kaplan-Meier analysis was used to complete the overall survival and disease-free survival of the selected genes in the core module. Results. We identified 96 candidate targets of ZJC and 86 DEGs of LSCC, the latter including 50 upregulated genes and 36 downregulated genes. DEGs were obviously enriched in the following biological functions: extracellular structure organization, the extracellular matrix organization, and endodermal cell differentiation. The $60 \mathrm{key}$ targets from the core network were enriched in the signal pathways including transcriptional misregulation cancer, cell cycle, and so on. We found that LSCC patients with high expression of HIST1H3J, HIST1H3F, and ITGA4 had worse overall survival, while higher expression of NTRK1, COPS5, HIST1H3A, and HIST1H3G had significantly worse disease-free survival. Conclusion. It suggested that the interaction between ZJC and LSCC was related to the signal pathways of transcriptional misregulation cancer and cell cycle, revealing that it may be the mechanism of ZJC in the treatment of LSCC.

\section{Introduction}

Laryngeal squamous cell carcinoma (LSCC) is the most common malignancy of the larynx, and its clinical manifestations are hoarseness, stridor, dyspnea, and even dysphagia $[1,2]$. Disappointingly, despite various technologies such as surgery, laser therapy, and chemoradiation have advanced recently, and the survival rate has not improved because of a high rate of recurrence and metastasis $[3,4]$. Therefore, in order to improve survival rates of the patients, there is an urgent need for effective treatment.

An increasing number of studies confirmed that traditional Chinese medicine (TCM) including multiple ingredients and targets play a critical role in the treatment of cancer. Zao-Jiao-Ci (ZJC), also known as Gleditsia sinensis, is a traditional Chinese medicine with a variety of bioactivities, especially antitumor activity, which has been widely used in clinic [5]. It was investigated that the ethanol extract of Gleditsia sinensis (EEGS) could suppress the growth of human colon cancer HCT116 cells in vitro and in vivo [6]. The extract of Gleditsia sinensis fruit performed inhibitory effects on esophageal squamous cell carcinoma (ESCC) cells, breast cancer MCF-7 cells, hepatoblastoma HepG2 cells, and so on $[7,8]$. However, there is no study to investigate the anticancer effect of Gleditsia sinensis on LSCC, and the mechanism remains unclear. 
Network pharmacology has exhibited specific utility in analyzing multicomponent and multitarget, consistent with the therapy hypothesis of complex diseases. By constructing a multilevel, multifaceted network model comprised of components, targets, pathways, and diseases, we can investigate TCM in the treatment of disease involved in the regulation of a variety of signaling pathways, key targets taxa, and biological process analysis, aiming to reveal the mechanism from the molecular level [9].

In this study, we used network pharmacology to investigate whether ZJC exerts anticancer effects on LSCC based on the GEO microarray dataset. And through the pathway enrichment analysis of the interaction targets between differentially expressed gene (DEGs) of LSCC and key node targets of ZJC, we further predicted the therapeutic mechanism of ZJC on LSCC. To our knowledge, this study is the first to explore the efficacy and mechanism of ZJC on LSCC, providing theoretical support and directions for further basic research.

\section{Methods}

2.1. Active Ingredients Screening and Targets Prediction for ZJC. Through the Traditional Chinese Medicine Systems Pharmacology Database and Analysis Platform (TCMSP), all components of ZJC could be found by searching the term "Zao-Jiao-Ci." We set oral bioavailability $(\mathrm{OB})>30 \%$ and drug-likeness (DL) $>0.18$ as screening conditions supported by the published literatures to obtain the final active ingredients $[10,11]$. PharmMapper server is the first webserver for potential drug targets identification through large-scale reverse pharmacophore mapping strategy [12]. The MOL structure of active ingredients provided by TCMSP was input into PharmMapper server (http://lilab.ecust.edu.cn/ pharmmapper/) to get the targets of the pharmacophore model. The first 15 targets sorted by the fit score were seemed as candidate targets of ZJC.

2.2. Active Ingredient-Target PPI Network Construction. To explore the association between ingredients and targets, we established an interaction network. Cytoscape 3.7.2, one of the most favorite open-source software tools, provides visually biomedical interaction networks composed of protein, gene, and other types of interactions. It was used to develop an active ingredient-target PPI network to visualize the relationship between the active ingredients and their targets of ZJC.

2.3. GEO Data Collection and DEGs Identification. The original data series GSE84957 was downloaded from the Gene Expression Omnibus (GEO) microarray dataset, which contained gene expression profiles of 18 tissue samples (9 LSCC tumor tissues and 9 normal tissues). The $R$ language was used to process the original data sets, and the RMA algorithm of Affy software package was used to perform background correction and quartile standardization of the expression matrix. The gene ID, the gene probe name of the expression matrix, was replaced by the gene symbol provided by the GPL17843 Agilent-042818 Human lncRNA Microarray 8_24_v2 platform, and the average value of multiple probes for the same gene was used for analysis. Limma package was used to identify the significant differentially expressed genes (DEGs) according to $P<0.01, \mid \log 2$ (FC) $\mid>3$.

The screened DEGs were mapped into a volcano map using the $R$ language heatmap package for intuitive vision; finally, the clusterProfiler package was used to carry out GO enrichment analysis and KEGG pathway enrichment analysis for DEGs.

2.4. PPI Network Construction. BisoGenet plugin, comprising of six available PPI databases (the Biological General Repository for Interaction Datasets (BioGRID), Biomolecular Interaction Network Database (BIND), Molecular Interaction Database (MINT), Human Protein Reference Database (HPRD), and Database of Interacting Proteins (DIP)), was used to build the PPI network for DEGs and candidate target genes, respectively [13]. Then, the merged network was conducted for the two PPI networks. We filtered the output nodes with degrees of freedom greater than 2 times the median of all nodes according to the indicators of degree and betweenness centrality. Then, a core PPI network was constructed using CytoNCA, a Cytoscape plugin. The ClueGO plugin was used for the KEGG signaling pathway enrichment analysis. $P<0.01$ was taken as the inclusion standard for pathway items. The results of enrichment analysis were presented in the form of the pie chart and nodes.

2.5. Cluster of the Core PPI Network. The MCODE plugin in Cytoscape software was used to screen the highly clustered important modules in the core PPI network. We set the parameters as degree cutoff $=2$ and $\kappa$-core $=2$ and conducted KEGG signaling pathway enrichment analysis for the most significantly clustered modules.

2.6. Gene Expression Data of the Core Cluster for LSCC. The correlation between survival rates of LSCC patients (disease-free survival rate and overall survival rate) and the gene expression levels (NTRK1, COPS5, HIST1H3A, HIST1H3G, HIST1H3J, HIST1H3J, HIST1H3F, and ITGA4) were calculated using GEPIA online database (http://GEPIA. cancer pku.cn/) [14].

\section{Results}

3.1. Active Ingredients and Targets of ZJC. Searching for all the reported components in the TCMSP database, 30 active ingredients of ZJC were collected. Sequentially, only 11 active ingredients were retained which conformed to $\mathrm{OB}>30 \%$ and $\mathrm{DL}>0.18$, such as fisetin, fustin, flavanone, and kaempferol (Table 1). Then, 96 candidate targets of the above 11 active components were obtained after the duplicate targets were excluded. 
TABLE 1: The active ingredients in ZJC.

\begin{tabular}{lccr}
\hline No. & Component & OB (\%) & DL \\
\hline 1 & Fisetin & 52.60 & 0.24 \\
2 & Fustin & 50.91 & 0.24 \\
3 & $(-)$-Taxifolin & 60.51 & 0.27 \\
4 & Flavanone & 41.35 & 0.24 \\
5 & Beta-sitosterol & 36.91 & 0.75 \\
6 & Sitosterol & 36.91 & 0.75 \\
7 & Kaempferol & 41.88 & 0.24 \\
8 & Stigmasterol & 43.83 & 0.76 \\
9 & Stigmast-4-ene-3,6-dione & 39.12 & 0.79 \\
10 & Ent-epicatechin & 48.96 & 0.24 \\
11 & Quercetin & 46.43 & 0.28 \\
\hline
\end{tabular}

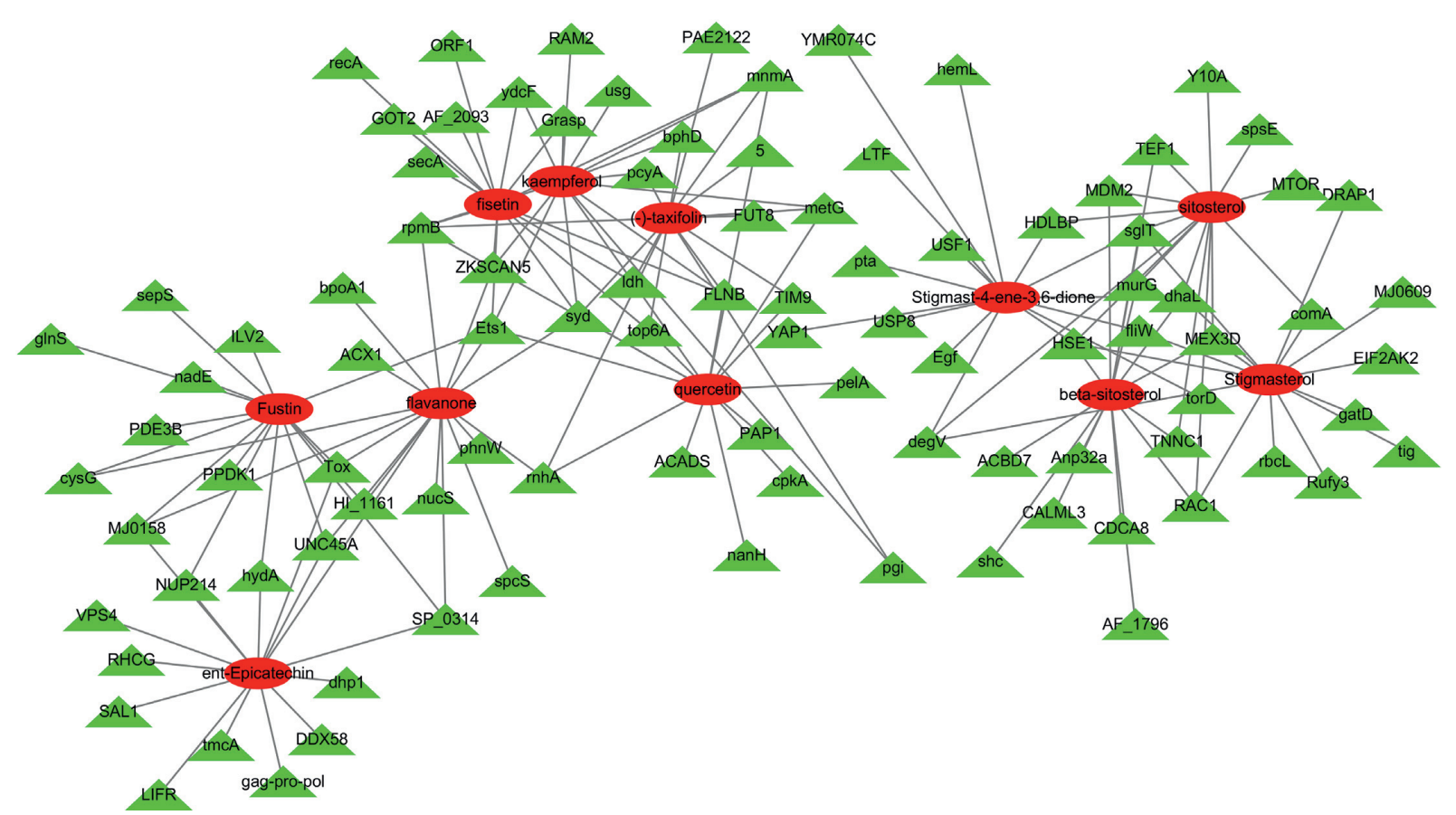

FIgURE 1: Active ingredients-targets network of ZJC. The blue circles represent the active ingredients, the red diamonds represent the targets, and the interaction between the two is represented by gray edges.

3.2. Active Ingredients-Targets PPI Network Construction. A PPI network of the active components and relevant targets, containing 107 nodes and 165 edges, was constructed by the network graphing tool Cytoscape 3.7.2. The 11 active ingredients could be connected with multiple targets, respectively, and each target also could be connected with multiple active ingredients, which directly demonstrated the relationship between active ingredients and targets of ZJC (Figure 1).

3.3. LSCC Differentially Expressed Genes (DEGs). By analyzing the gene chip of GSE84957, a total of 81 genes with significant different expression of the LSCC tissues compared with adjacent nonneoplastic tissues were obtained, among which 50 genes were upregulated and 31 genes were downregulated in tumor tissues (Table 2; Figure 2).
3.4. GO Enrichment Analysis and KEGG Pathway Analysis for DEGs. GO enrichment analysis was used to explore the molecular mechanism of DEGs. The results were given as follows: (i) in the BP category, DEGs were mostly enriched in the extracellular structure organization, the extracellular matrix organization, endodermal cell differentiation, endoderm formation, and endoderm development; (ii) in the category of CC, DEGs were mainly enriched in the extracellular matrix, collagen-containing extracellular matrix, endoplasmic reticulum lumen, collagen trimer, and extracellular matrix component; (iii) in the MF category, extracellular matrix structural constituent, cytokine activity, and receptor ligand activity were selected for main MF. The results of KEGG pathway analysis showed that ECM-receptor interaction, protein digestion and absorption, focal adhesion, Staphylococcus aureus infection, and viral protein interaction with cytokine and cytokine receptor were the major pathways involved in DEGs (Figure 3). 
TABLE 2: Differently expressed genes from GSE84957.

\begin{tabular}{|c|c|c|c|c|c|c|}
\hline GeneSample & $\log \mathrm{FC}$ & AveExpr & $t$ & $P$ value & Adj. $P$ value & $\mathrm{B}$ \\
\hline CST1 & 4.255518022 & 7.417759878 & 14.09776157 & $1.49 E-11$ & $3.71 E-07$ & 15.51198443 \\
\hline XLOC_004426 & 3.044228211 & 3.370726783 & 11.59258332 & $4.32 E-10$ & $3.61 E-06$ & 12.75595229 \\
\hline MMP11 & 3.903325333 & 6.986877144 & 11.58573097 & $4.36 E-10$ & $3.61 E-06$ & 12.74750381 \\
\hline GPRIN1 & 3.857745044 & 5.653573411 & 11.29565743 & $6.67 E-10$ & $4.15 E-06$ & 12.38486185 \\
\hline COL7A1 & 3.191737644 & 9.258242733 & 10.78042002 & $1.45 E-09$ & $7.22 E-06$ & 11.71597451 \\
\hline FAM3D & -5.792545067 & 7.2950313 & -10.09792 & $4.24 E-09$ & $1.51 E-05$ & 10.77877059 \\
\hline LRP12 & 3.580821556 & 4.005304867 & 9.316160682 & $1.54 E-08$ & $4.26 E-05$ & 9.629111691 \\
\hline CTHRC1 & 4.740806433 & 7.128087172 & 9.196467428 & $1.89 E-08$ & $4.30 E-05$ & 9.445581444 \\
\hline LOC100506027 & 4.143287578 & 4.568671189 & 8.999347488 & $2.66 E-08$ & $4.97 E-05$ & 9.138857621 \\
\hline TJP3 & -3.364634633 & 7.834813517 & -8.904023211 & $3.14 E-08$ & $4.97 E-05$ & 8.9885142 \\
\hline IGFBP3 & 3.604138167 & 10.55244692 & 8.893742313 & $3.20 E-08$ & $4.97 E-05$ & 8.972220302 \\
\hline ARSI & 3.152646867 & 3.986923189 & 8.298478812 & $9.26 E-08$ & 0.000121313 & 8.002153147 \\
\hline TMEM158 & 3.718106033 & 4.697089117 & 8.222894187 & $1.06 E-07$ & 0.000132353 & 7.875178695 \\
\hline PLAUR & 3.027472033 & 7.824632761 & 8.051498015 & $1.46 E-07$ & 0.000165132 & 7.584034636 \\
\hline MSR1 & 3.918547922 & 4.497970906 & 7.705672 & $2.80 E-07$ & 0.000248682 & 6.98290287 \\
\hline TGFBI & 3.415596633 & 9.235072794 & 7.534242043 & $3.89 E-07$ & 0.000311941 & 6.678084284 \\
\hline SPP1 & 4.853097222 & 7.318721011 & 7.296283387 & $6.17 E-07$ & 0.000415016 & 6.247440183 \\
\hline CYP4B1 & -3.938406289 & 5.174530178 & -7.145413133 & $8.31 E-07$ & 0.000492716 & 5.96986977 \\
\hline SH3BGRL2 & -4.071528089 & 6.438326422 & -7.12999436 & $8.57 E-07$ & 0.000495582 & 5.941304558 \\
\hline LOC100653149 & 3.268119356 & 5.443807811 & 7.078046014 & $9.50 E-07$ & 0.000525005 & 5.844794163 \\
\hline MMP1 & 4.862459922 & 6.783056994 & 7.002232691 & $1.10 E-06$ & 0.000590601 & 5.70320204 \\
\hline TM4SF19 & 4.052795678 & 5.81647465 & 6.87435807 & $1.43 E-06$ & 0.000696569 & 5.462380919 \\
\hline COL5A2 & 3.504323078 & 7.584409283 & 6.759032897 & $1.80 E-06$ & 0.000815742 & 5.243053839 \\
\hline HOXD11 & 3.550990856 & 5.087563172 & 6.54672159 & $2.78 E-06$ & 0.001117349 & 4.834020589 \\
\hline CXCL11 & 4.762321211 & 4.652769394 & 6.519758265 & $2.94 E-06$ & 0.00115171 & 4.781590785 \\
\hline LOC100652832 & 3.253860656 & 6.465006439 & 6.502526246 & $3.05 E-06$ & 0.00115171 & 4.748026804 \\
\hline CXCL10 & 4.519192844 & 5.640273644 & 6.498769364 & $3.07 E-06$ & 0.00115171 & 4.740703422 \\
\hline XLOC_006053 & -3.635899633 & 9.156396294 & -6.475756406 & $3.22 E-06$ & 0.00115171 & 4.69579809 \\
\hline KRT17 & 4.404712111 & 11.51599561 & 6.464235069 & $3.30 E-06$ & 0.00115171 & 4.673287016 \\
\hline PTHLH & 4.098820033 & 7.949679894 & 6.341212465 & $4.26 E-06$ & 0.001315184 & 4.431701824 \\
\hline CXCL12 & -3.476515767 & 8.330393117 & -6.24092991 & $5.26 E-06$ & 0.001422596 & 4.233143024 \\
\hline XLOC_12_006021 & 4.372459256 & 11.50269782 & 6.229963809 & $5.38 E-06$ & 0.001424781 & 4.211342414 \\
\hline COL8A 1 & 3.211121667 & 6.060922078 & 6.177430972 & $6.01 E-06$ & 0.001526438 & 4.106669064 \\
\hline CLCA4 & -5.919341656 & 8.217143061 & -6.126082361 & $6.70 E-06$ & 0.001661254 & 4.003977039 \\
\hline HMGA2 & 3.706329122 & 3.703429261 & 6.117033971 & $6.83 E-06$ & 0.00166615 & 3.985842669 \\
\hline WISP1 & 3.021981556 & 5.595450789 & 6.039266244 & $8.06 E-06$ & 0.001838778 & 3.829512486 \\
\hline NRG1 & 3.430506311 & 6.615773067 & 6.013403137 & $8.51 E-06$ & 0.001907796 & 3.777336092 \\
\hline ANKRD20A9P & -3.027104311 & 6.964110233 & -5.991259577 & $8.92 E-06$ & 0.001947336 & 3.732590438 \\
\hline GCNT3 & -3.483360222 & 5.808183467 & -5.968076797 & $9.38 E-06$ & 0.001965485 & 3.685672938 \\
\hline MYOC & -3.290936644 & 3.378123278 & -5.964261662 & $9.45 E-06$ & 0.001965485 & 3.677944815 \\
\hline ODZ2 & 3.030850211 & 7.342800339 & 5.955698768 & $9.63 E-06$ & 0.001965485 & 3.660592194 \\
\hline DNAPTP3 & 4.162512589 & 8.355008817 & 5.948057503 & $9.79 E-06$ & 0.001979224 & 3.645098841 \\
\hline FUT3 & -3.452809133 & 7.136305 & -5.832221646 & $1.25 E-05$ & 0.00221345 & 3.409270286 \\
\hline CRNN & -6.524733278 & 9.72241585 & -5.821941297 & $1.28 E-05$ & 0.002227034 & 3.388254529 \\
\hline CFD & -3.520037456 & 9.369048272 & -5.800510125 & $1.34 E-05$ & 0.002286888 & 3.344398953 \\
\hline COL1A2 & 3.096446333 & 11.78684306 & 5.800402532 & $1.34 E-05$ & 0.002286888 & 3.34417863 \\
\hline CXCL9 & 3.067348622 & 6.615663178 & 5.759709415 & $1.47 E-05$ & 0.002369549 & 3.260741185 \\
\hline PDPN & 3.170275733 & 10.0438188 & 5.709284988 & $1.64 E-05$ & 0.00252911 & 3.157054467 \\
\hline INHBA & 3.099887078 & 5.809753317 & 5.656043661 & $1.84 E-05$ & 0.002640463 & 3.04722486 \\
\hline MMP12 & 4.162521167 & 3.736549417 & 5.638864584 & $1.91 E-05$ & 0.002687547 & 3.011710913 \\
\hline SCARA5 & -3.861003467 & 7.106386856 & -5.634806253 & $1.92 E-05$ & 0.002687547 & 3.003315853 \\
\hline COL4A1 & 3.293993722 & 11.03886292 & 5.623430739 & $1.97 E-05$ & 0.002709232 & 2.979773593 \\
\hline COL5A1 & 3.205179622 & 8.674301744 & 5.604407653 & $2.05 E-05$ & 0.00279295 & 2.940368581 \\
\hline ANKRD20A5P & -3.118826611 & 5.357065783 & -5.574210889 & $2.19 E-05$ & 0.002873117 & 2.877727019 \\
\hline TNXB & -3.390147544 & 8.386793894 & -5.46437267 & $2.79 E-05$ & 0.003345526 & 2.648949986 \\
\hline CCDC25 & -3.340572133 & 7.218539933 & -5.461095079 & $2.81 E-05$ & 0.003345526 & 2.6421014 \\
\hline FN1 & 3.580535444 & 11.63254756 & 5.427958008 & $3.02 E-05$ & 0.003525959 & 2.572791248 \\
\hline MAL & -6.360331133 & 8.9132381 & -5.380498508 & $3.36 E-05$ & 0.003726774 & 2.473306046 \\
\hline FBN2 & 3.702098767 & 4.647001128 & 5.317540391 & $3.86 E-05$ & 0.003963698 & 2.340945176 \\
\hline KRT4 & -5.783585056 & 12.44990214 & -5.27501498 & $4.24 E-05$ & 0.004304541 & 2.251297748 \\
\hline
\end{tabular}


TABLE 2: Continued.

\begin{tabular}{|c|c|c|c|c|c|c|}
\hline GeneSample & $\log \mathrm{FC}$ & AveExpr & $t$ & $P$ value & Adj. $P$ value & B \\
\hline MSC & 3.021481811 & 5.778950494 & 5.273063535 & $4.25 E-05$ & 0.004304541 & 2.247179302 \\
\hline MYZAP & -3.966621 & 6.975986556 & -5.218924365 & $4.80 E-05$ & 0.00453758 & 2.132761995 \\
\hline CHI3L1 & 3.447755856 & 8.454925183 & 5.212706409 & $4.86 E-05$ & 0.004565936 & 2.119601631 \\
\hline XLOC_008370 & -4.086801056 & 5.071876417 & -5.195774385 & $5.05 E-05$ & 0.004618685 & 2.083744902 \\
\hline AMY1C & -3.066162922 & 4.171807861 & -5.181124682 & $5.22 E-05$ & 0.004702673 & 2.052698071 \\
\hline FAM107 A & -3.366929044 & 6.613174211 & -5.153177492 & $5.55 E-05$ & 0.004829406 & 1.993410827 \\
\hline CA9 & 4.004719489 & 4.832498267 & 5.105740988 & $6.17 E-05$ & 0.005199505 & 1.89260426 \\
\hline SFI1 & -3.172654489 & 7.898643289 & -5.038975245 & $7.16 E-05$ & 0.005657681 & 1.75036129 \\
\hline KRT24 & -4.307497756 & 6.663276378 & -4.903497209 & $9.71 E-05$ & 0.006802832 & 1.460515802 \\
\hline FAM3B & -3.002864178 & 7.472917967 & -4.851035631 & 0.000109236 & 0.007385034 & 1.347873623 \\
\hline PSCA & -4.424161267 & 6.177034822 & -4.836697273 & 0.000112824 & 0.007525305 & 1.317050516 \\
\hline TREM1 & 3.132156567 & 5.329140306 & 4.802412753 & 0.000121894 & 0.007755997 & 1.243287728 \\
\hline MMP7 & 4.0077695 & 8.433879583 & 4.797447382 & 0.000123267 & 0.007808567 & 1.232597743 \\
\hline XLOC_12_007931 & -3.335940244 & 10.48608082 & -4.789159173 & 0.000125595 & 0.007875182 & 1.214750103 \\
\hline KRT6B & 4.076616322 & 9.703940506 & 4.773184049 & 0.000130209 & 0.007978995 & 1.180336064 \\
\hline SERPINE1 & 3.5038576 & 7.4466057 & 4.752919626 & 0.00013631 & 0.008152053 & 1.136656679 \\
\hline ABCA8 & -3.2918408 & 5.542601178 & -4.749840302 & 0.000137262 & 0.008169717 & 1.130016856 \\
\hline SNX31 & -3.294362333 & 3.714450967 & -4.727065435 & 0.000144517 & 0.008363625 & 1.080888859 \\
\hline KRT16 & 3.956891967 & 10.74006252 & 4.71119429 & 0.000149801 & 0.008567571 & 1.046633089 \\
\hline RSAD2 & 3.410952911 & 5.3110705 & 4.667343136 & 0.000165442 & 0.009086152 & 0.951904596 \\
\hline CEACAM5 & -3.982757711 & 9.060092478 & -4.649201732 & 0.000172387 & 0.009343839 & 0.912681384 \\
\hline
\end{tabular}

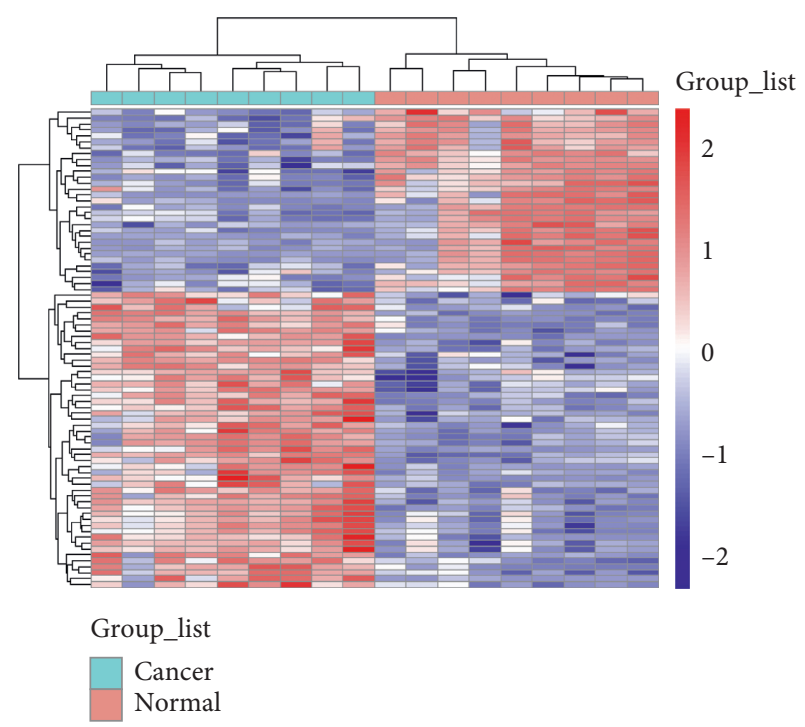

(a)

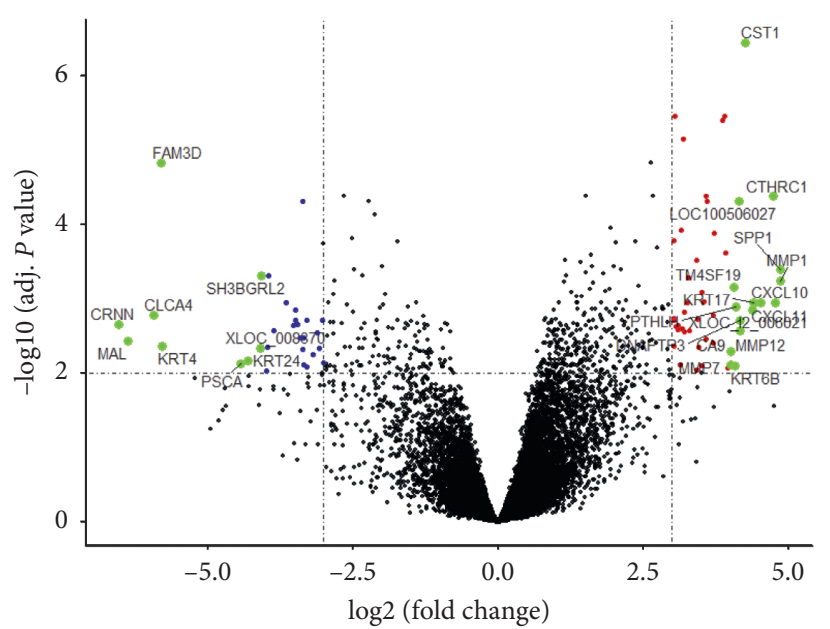

(b)

Figure 2: Volcano plot of gene expression and heatmap of DEGs. (a) The rows represent genes and the columns represent samples. The first 9 columns are tumor samples and the last 9 columns are normal samples. Red represents high gene expression, and blue represents low gene expression. (b) The red, blue, and green dots represent the differentially expressed genes between the tumor tissues and normal tissues of LSCC, among which the red represents the upregulated genes in the tumor tissues, the blue represents the downregulated genes, and the green represents the genes of $\log \mathrm{FC}>4$, while the black represents the insignificantly different genes.

3.5. PPI Network Construction and Key Targets Screening. A PPI network based on the targets of ZJC active ingredients was constructed. It showed that ZJC had direct or indirect correlation with the 1572 targets, and there were 29,098 interconnections between these targets. At the same time, the PPI network was mapped for DEGs, and 2,262 targets were directly or indirectly related to LSCC, with 50,181 interconnections between these targets. Then, the intersections of the two PPI networks were used to construct a merged network with 510 nodes and 10950 edges (Figures 4(a)-4(c)). Furthermore, we analyzed the topological properties of the nodes in the merged network of the protein interactions to find the key nodes. Finally, 60 key nodes were identified through the network topology analysis (Figure 4(d) and Table 3).

3.6. KEGG Pathway Analysis and Main Module of the Core PPI Network. The KEGG signaling pathways analysis suggested that 60 key targets were mainly enriched in cell cycle, central 


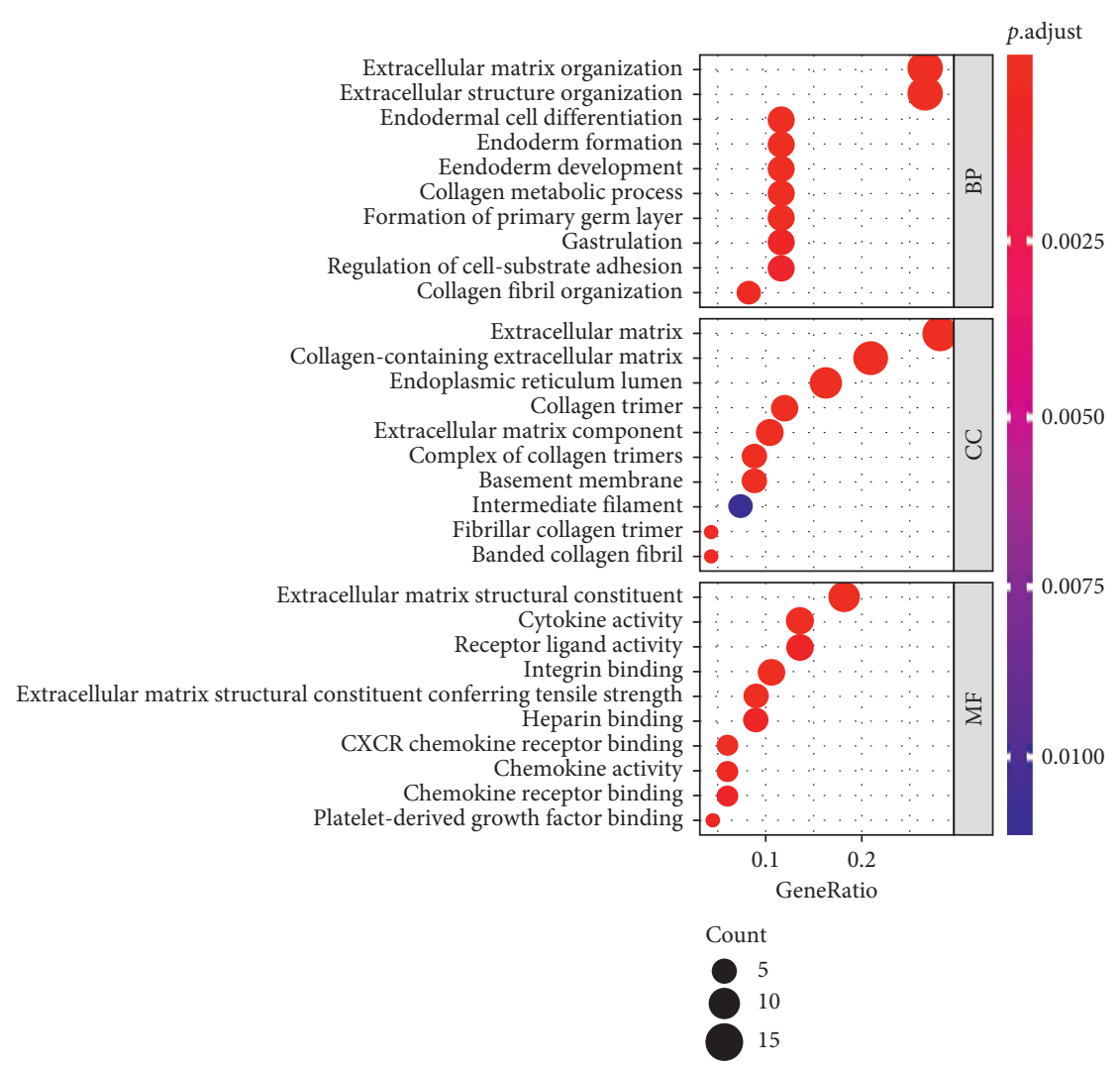

(a)

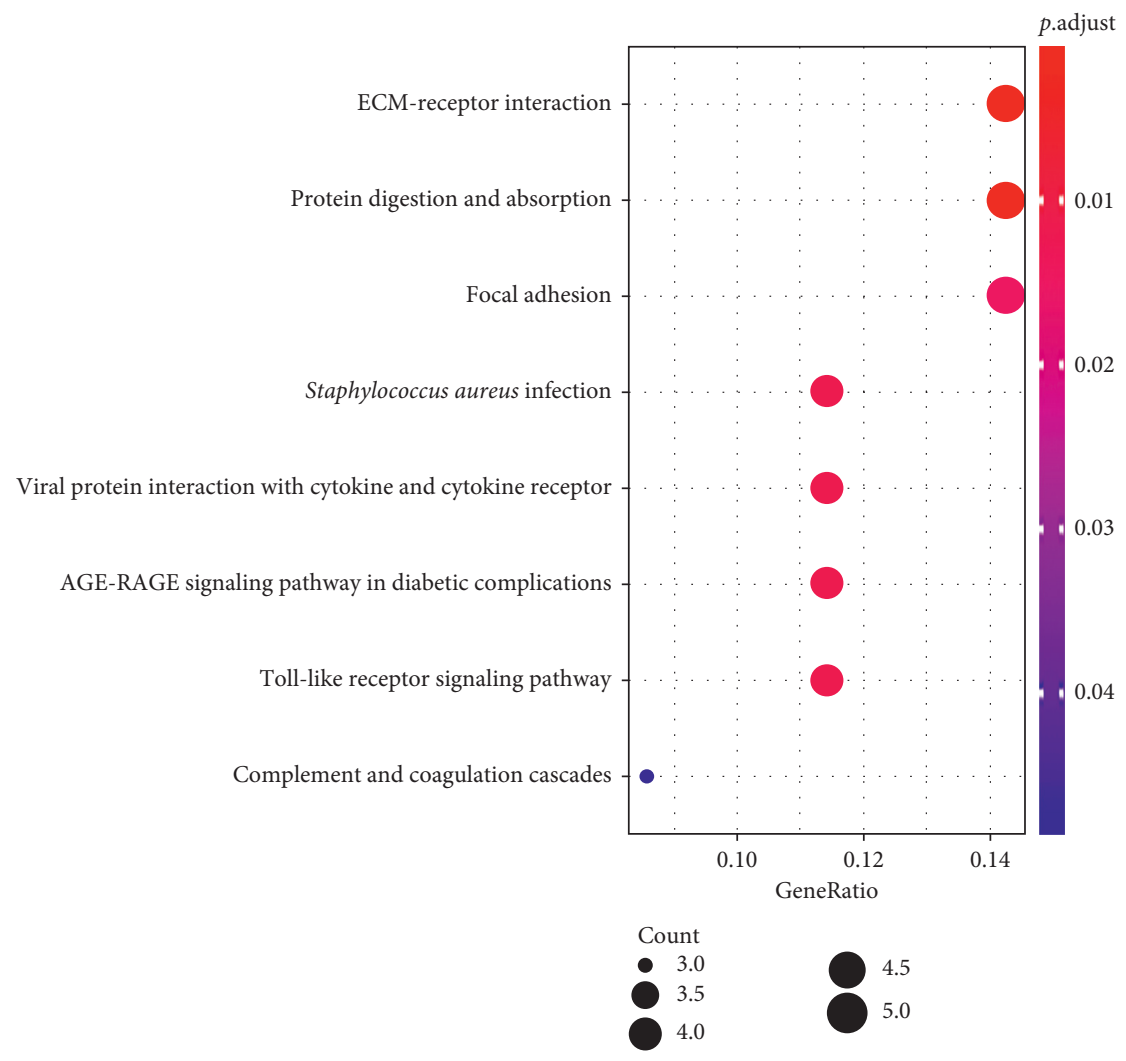

(b)

FIGURE 3: GO enrichment analysis and KEGG pathways analysis on DEGs. (a) GO enrichment analysis: the top 10 terms of biological process, cellular component, and molecular function with $P<0.05$. (b) KEGG pathways analysis: the top 3 terms of KEGG enrichment pathway with $P<0.05$. 


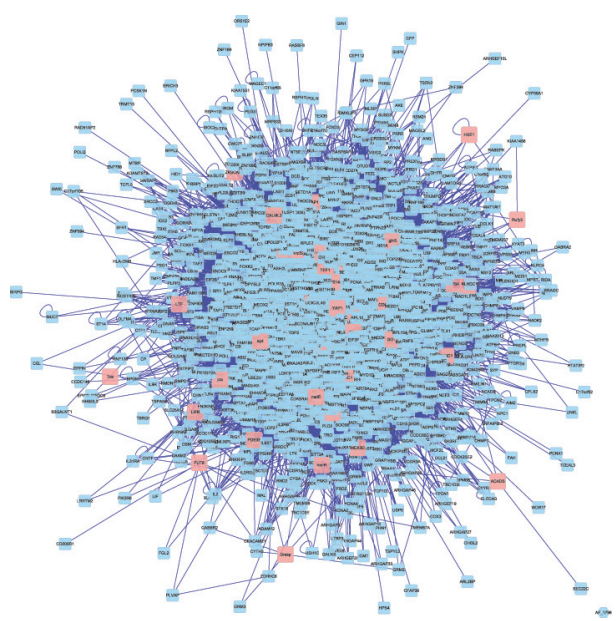

(a)

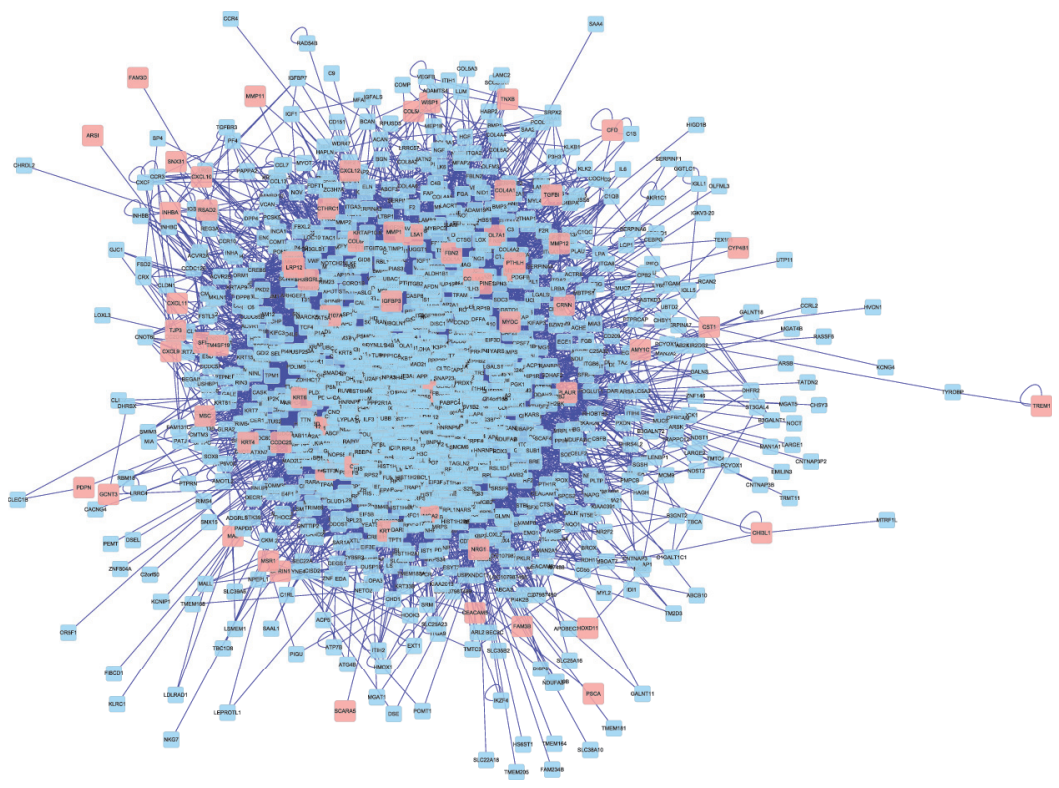

(b)

FIgURE 4: Continued. 


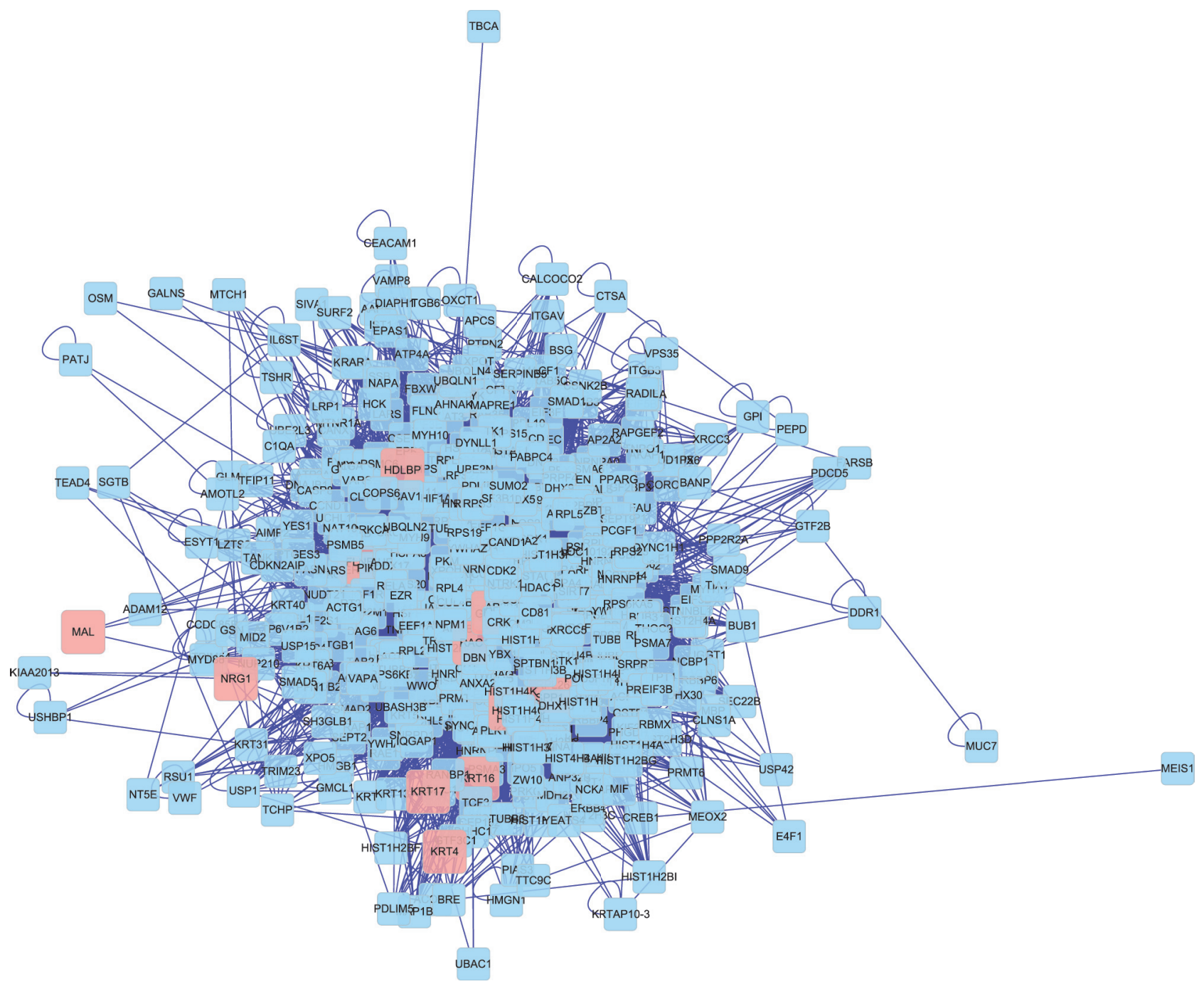

(c)

Figure 4: Continued. 


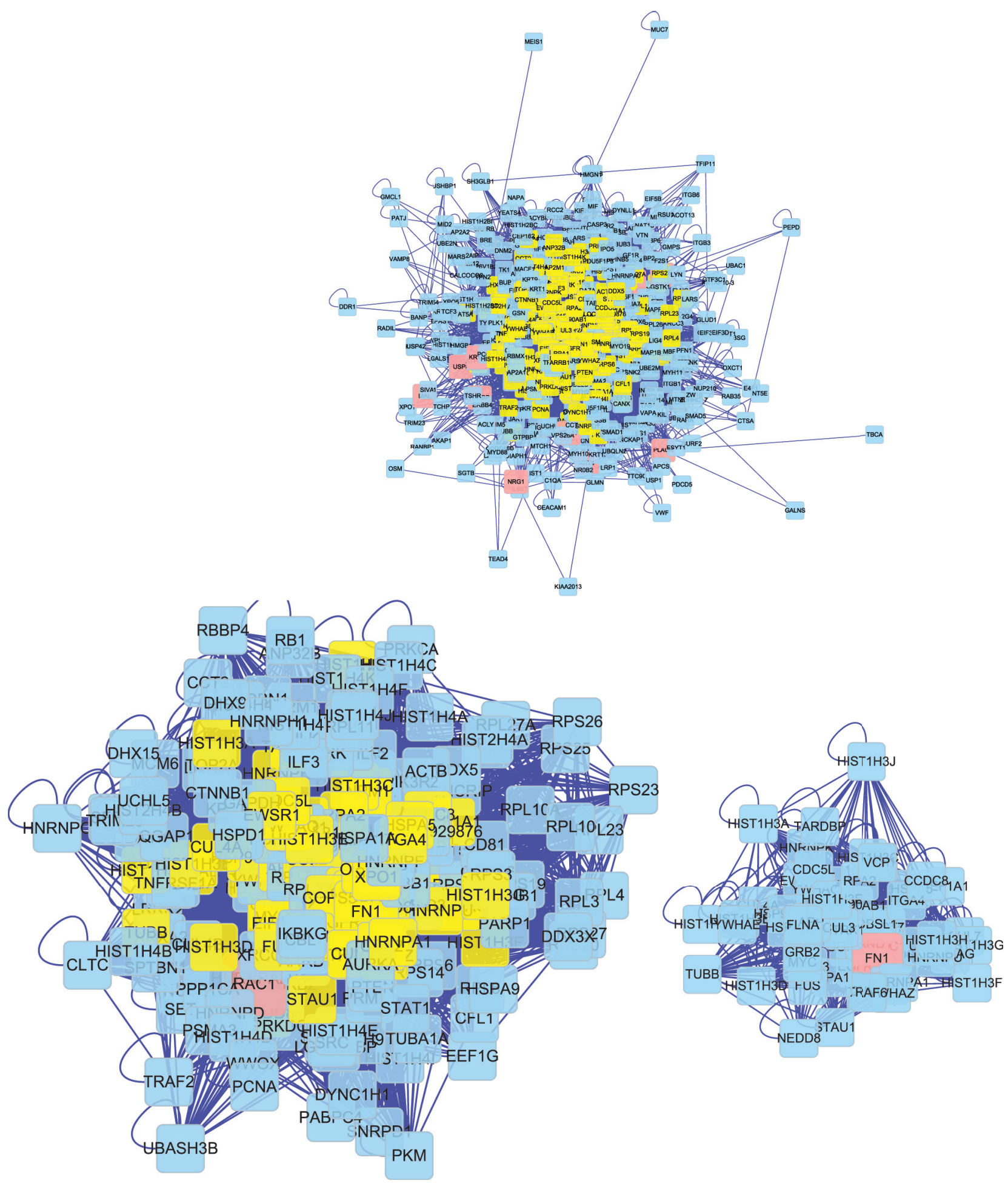

(d)

FIgURe 4: Identification of key targets for ZJC against LSCC. (a) PPI of ZJC targets. (b) PPI of DEGs in LSCC. (c) The intersections of the two PPI networks of ZJC and DEGs. (d) Topological screening of the interactive PPI network based on degree and betweenness centrality. The same type of the signaling pathway is represented by nodes of the same color, and the size of the node represents the significance of the signaling pathway. The higher the significance of the signaling pathway is, the larger the node is, indicating that the importance of the pathway is higher. 
TABLE 3: The topological properties of the 60 key nodes.

\begin{tabular}{|c|c|c|c|c|c|c|}
\hline Name (the key targets) & Degree & Betweenness & Betweenness centrality & Closeness & Closeness centrality & Topological coefficient \\
\hline YWHAZ & 155 & 3001.377571 & 0.01183919 & 0.585365854 & 0.58536585 & 0.14373835 \\
\hline YWHAG & 87 & 816.3912445 & 0.00322033 & 0.537313433 & 0.53731343 & 0.16424877 \\
\hline MCM5 & 101 & 1080.133008 & 0.00426068 & 0.542518837 & 0.54251884 & 0.14457089 \\
\hline STAU1 & 114 & 1407.485881 & 0.00555195 & 0.550218341 & 0.55021834 & 0.13468492 \\
\hline ITGA4 & 208 & 7021.25165 & 0.02769593 & 0.616891065 & 0.61689106 & 0.10712371 \\
\hline APP & 125 & 5666.777349 & 0.02235309 & 0.557522124 & 0.55752212 & 0.10929128 \\
\hline CUL3 & 211 & 7900.538977 & 0.03116436 & 0.626865672 & 0.62686567 & 0.1083315 \\
\hline CUL2 & 121 & 2193.490854 & 0.00865241 & 0.556906077 & 0.55690608 & 0.1311238 \\
\hline CUL4A & 84 & 1104.388869 & 0.00435636 & 0.519052523 & 0.51905252 & 0.14961657 \\
\hline CUL1 & 158 & 2737.270054 & 0.0107974 & 0.579976985 & 0.57997699 & 0.12774443 \\
\hline COPS5 & 173 & 4596.081368 & 0.01812964 & 0.592941176 & 0.59294118 & 0.11812865 \\
\hline YWHAQ & 116 & 2340.92677 & 0.00923399 & 0.5532382 & 0.5532382 & 0.13955671 \\
\hline EEF1A1 & 111 & 2303.865101 & 0.0090878 & 0.553846154 & 0.55384615 & 0.15726594 \\
\hline OBSL1 & 151 & 2614.051749 & 0.01031135 & 0.572077185 & 0.57207719 & 0.11367624 \\
\hline TARDBP & 99 & 902.4212542 & 0.00355968 & 0.540192926 & 0.54019293 & 0.14812918 \\
\hline HSPA8 & 102 & 1110.484051 & 0.0043804 & 0.549019608 & 0.54901961 & 0.1617004 \\
\hline HSPA5 & 124 & 2786.82878 & 0.01099289 & 0.56187291 & 0.56187291 & 0.15308876 \\
\hline HSP90AA1 & 115 & 3058.241745 & 0.0120635 & 0.558139535 & 0.55813953 & 0.13921995 \\
\hline EIF4A3 & 94 & 923.5252369 & 0.00364293 & 0.535031847 & 0.53503185 & 0.14405585 \\
\hline HSP90AB1 & 111 & 2024.466508 & 0.00798568 & 0.555066079 & 0.55506608 & 0.14751182 \\
\hline MYC & 88 & 1227.898615 & 0.00484355 & 0.536741214 & 0.53674121 & 0.11599255 \\
\hline CCDC8 & 145 & 2628.810161 & 0.01036957 & 0.568848758 & 0.56884876 & 0.11392955 \\
\hline HDAC1 & 84 & 1756.679856 & 0.00692938 & 0.525547445 & 0.52554745 & 0.11154264 \\
\hline RPA1 & 106 & 1443.910092 & 0.00569563 & 0.544864865 & 0.54486486 & 0.13668578 \\
\hline RPA2 & 98 & 1508.905701 & 0.00595201 & 0.541353383 & 0.54135338 & 0.13008096 \\
\hline HNRNPA1 & 130 & 1803.567503 & 0.00711433 & 0.565656566 & 0.56565657 & 0.14709052 \\
\hline EGFR & 143 & 4724.066642 & 0.01863449 & 0.572727273 & 0.57272727 & 0.1103327 \\
\hline HIST1H3F & 86 & 437.5273205 & 0.00172586 & 0.529411765 & 0.52941176 & 0.14705882 \\
\hline UBC & 133 & 3538.655204 & 0.01395853 & 0.566929134 & 0.56692913 & 0.12972445 \\
\hline CUL7 & 174 & 4304.231567 & 0.01697841 & 0.594339623 & 0.59433962 & 0.10695547 \\
\hline HNRNPK & 90 & 914.3729176 & 0.00360682 & 0.535600425 & 0.53560043 & 0.17748918 \\
\hline TUBB & 85 & 1017.885462 & 0.00401514 & 0.537886873 & 0.53788687 & 0.16372149 \\
\hline HNRNPU & 106 & 2391.698718 & 0.00943426 & 0.547826087 & 0.54782609 & 0.15722622 \\
\hline CDK2 & 172 & 5391.386868 & 0.02126679 & 0.592941176 & 0.59294118 & 0.11382953 \\
\hline EWSR1 & 85 & 1514.316862 & 0.00597335 & 0.532206969 & 0.53220697 & 0.15183716 \\
\hline CDC5L & 113 & 2162.954989 & 0.00853196 & 0.550819672 & 0.55081967 & 0.13024984 \\
\hline NEDD8 & 86 & 533.4516789 & 0.00210425 & 0.528301887 & 0.52830189 & 0.14769424 \\
\hline HIST1H3A & 86 & 437.5273205 & 0.00172586 & 0.529411765 & 0.52941176 & 0.14705882 \\
\hline HIST1H3D & 86 & 437.5273205 & 0.00172586 & 0.529411765 & 0.52941176 & 0.14705882 \\
\hline TRAF6 & 100 & 2757.045931 & 0.01087541 & 0.538461538 & 0.53846154 & 0.09652163 \\
\hline HIST1H3C & 86 & 437.5273205 & 0.00172586 & 0.529411765 & 0.52941176 & 0.14705882 \\
\hline NPM1 & 156 & 3506.462925 & 0.01383155 & 0.58400927 & 0.58400927 & 0.14115178 \\
\hline HIST1H3E & 86 & 437.5273205 & 0.00172586 & 0.529411765 & 0.52941176 & 0.14705882 \\
\hline HIST1H3I & 86 & 437.5273205 & 0.00172586 & 0.529411765 & 0.52941176 & 0.14705882 \\
\hline HIST1H3G & 86 & 437.5273205 & 0.00172586 & 0.529411765 & 0.52941176 & 0.14705882 \\
\hline FUS & 109 & 1065.023899 & 0.00420108 & 0.551422319 & 0.55142232 & 0.157963 \\
\hline HIST1H3J & 86 & 437.5273205 & 0.00172586 & 0.529411765 & 0.52941176 & 0.14705882 \\
\hline HIST1H3H & 86 & 437.5273205 & 0.00172586 & 0.529411765 & 0.52941176 & 0.14705882 \\
\hline HIST1H3B & 86 & 437.5273205 & 0.00172586 & 0.529411765 & 0.52941176 & 0.14705882 \\
\hline NTRK1 & 270 & 16394.02825 & 0.06466766 & 0.673796791 & 0.67379679 & 0.09989618 \\
\hline FLNA & 89 & 1820.058448 & 0.00717938 & 0.537313433 & 0.53731343 & 0.15216314 \\
\hline FN1 & 307 & 27515.32665 & 0.10853658 & 0.708860759 & 0.70886076 & 0.09048518 \\
\hline SIRT7 & 128 & 2444.801055 & 0.00964373 & 0.5532382 & 0.5532382 & 0.11177503 \\
\hline GRB2 & 136 & 4533.847683 & 0.01788415 & 0.570135747 & 0.57013575 & 0.12538522 \\
\hline FBXO6 & 129 & 2240.28371 & 0.00883699 & 0.558139535 & 0.55813953 & 0.11844315 \\
\hline VCP & 103 & 1651.524516 & 0.00651458 & 0.544864865 & 0.54486486 & 0.14998166 \\
\hline CAND1 & 152 & 2355.429527 & 0.0092912 & 0.578645235 & 0.57864524 & 0.12845528 \\
\hline XPO1 & 106 & 2782.081388 & 0.01097416 & 0.543103448 & 0.54310345 & 0.12465874 \\
\hline MCM2 & 207 & 6633.522707 & 0.0261665 & 0.621454994 & 0.62145499 & 0.11887293 \\
\hline YWHAE & 93 & 1174.476977 & 0.00463283 & 0.537313433 & 0.53731343 & 0.16260551 \\
\hline
\end{tabular}




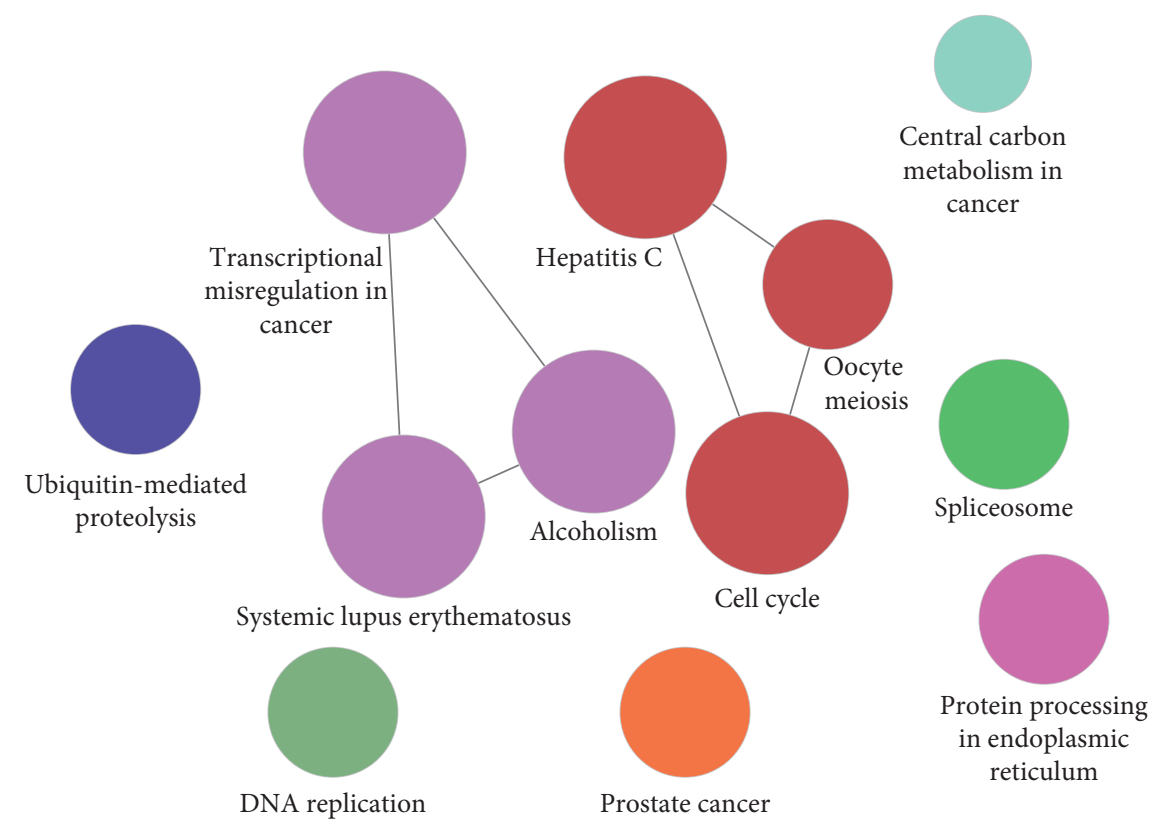

FIGURE 5: Vital terms of KEGG enrichment analysis of the key targets.

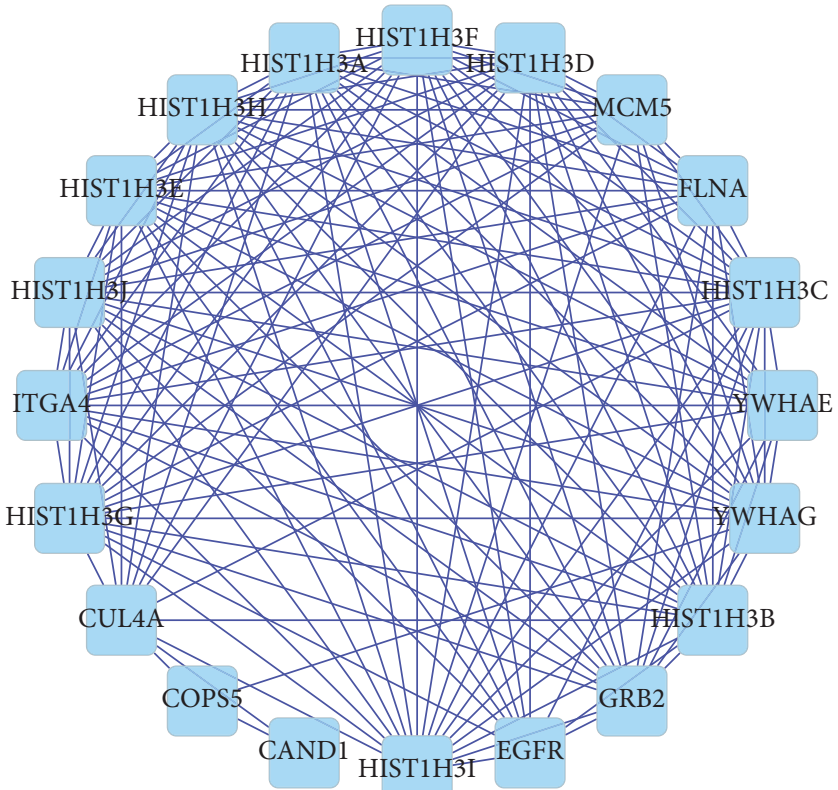

(a)

FIGURE 6: Continued. 


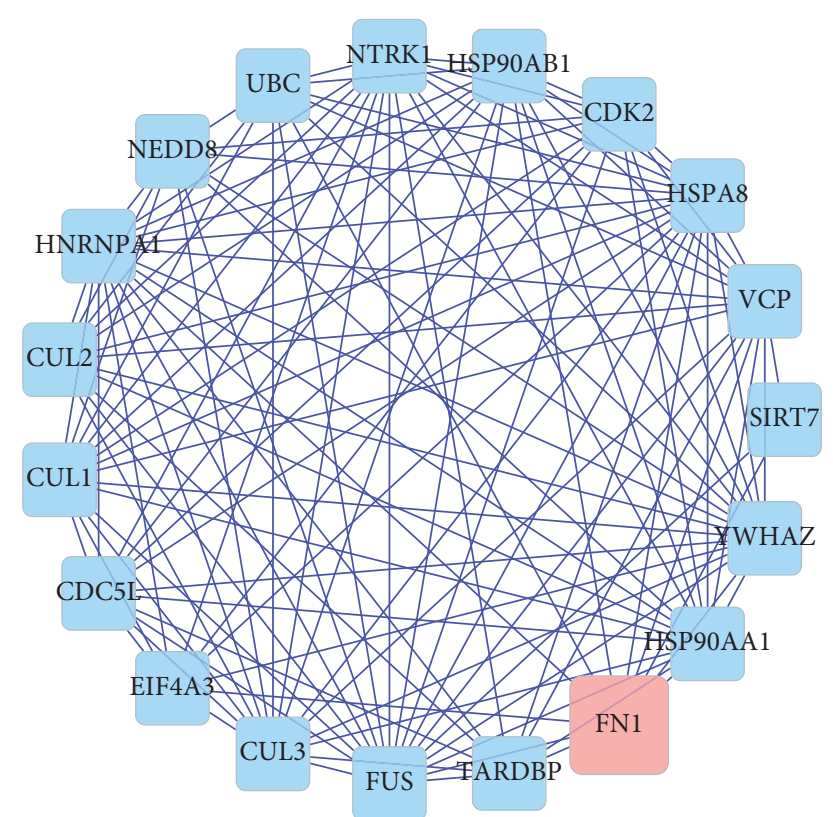

(b)

FIgURE 6: The two main modules of the core PPI network of ZJC against LSCC.

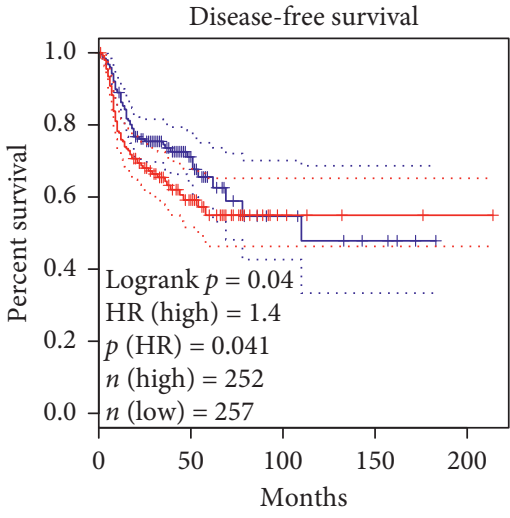

— Low HIST1H3A TPM

— High HIST1H3A TPM

(a)

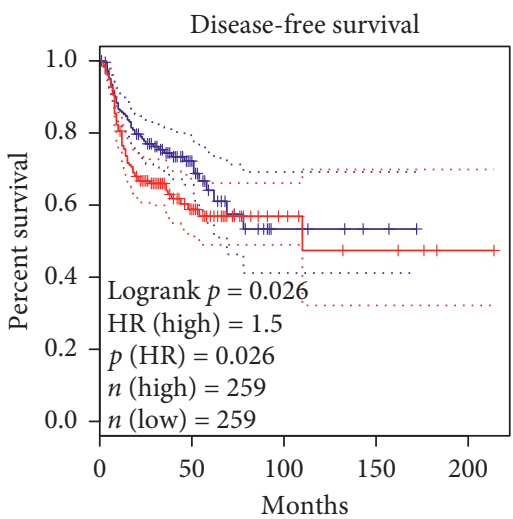

- Low COPS5 TPM

— High COPS5 TPM

(c)

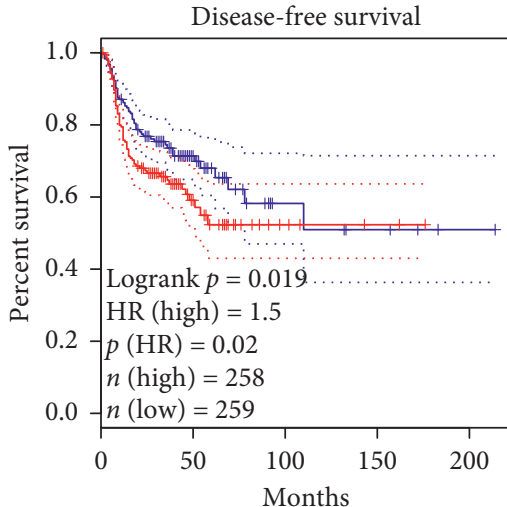

— Low HIST1H3G TPM

_ _ High HIST1H3G TPM

(b)

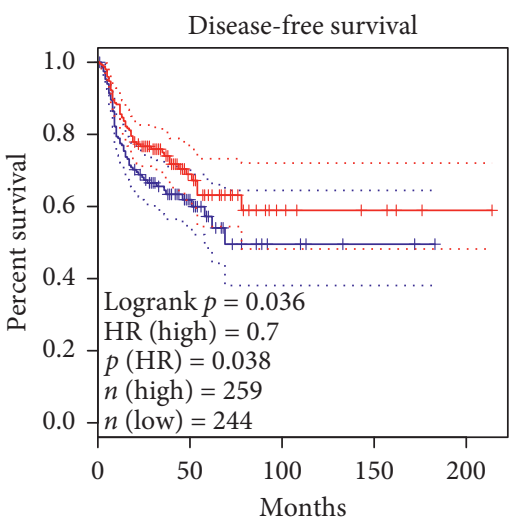

— Low NTRK1 TPM

- High NTRK1 TPM

(d)

Figure 7: Continued. 


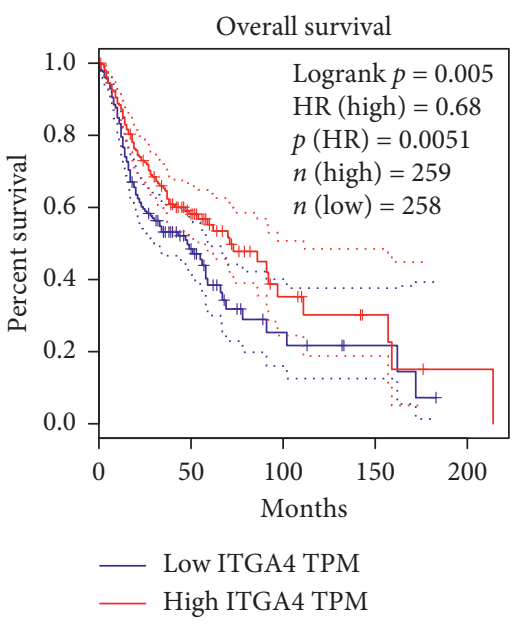

(e)

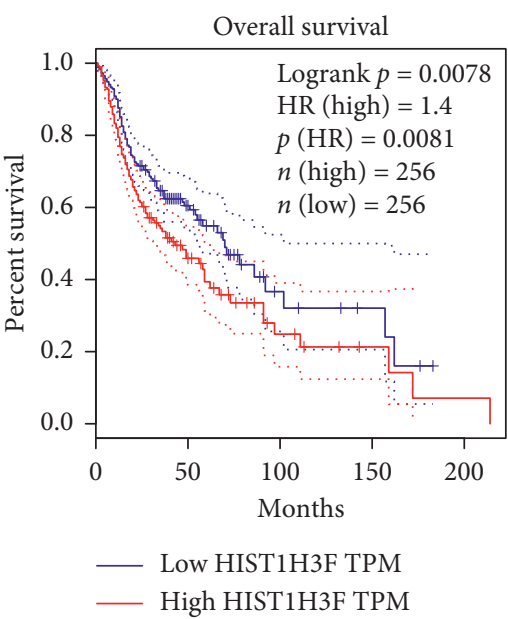

(f)

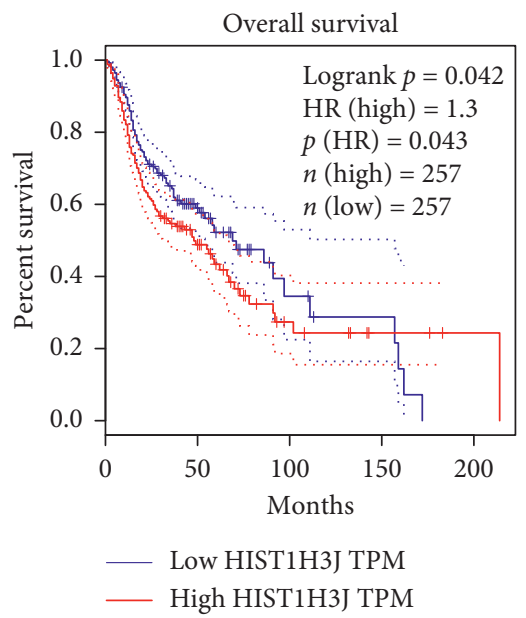

(g)

FIGURE 7: Disease-free survival analyses and overall survival analyses of LSCC. (a)-(d) Disease-free survival analyses of NTRK1, COPS5, HIST1H3A, and HIST1H3G about LSCC. (e)-(g) Overall survival analyses of HIST1H3J, HIST1H3F, and ITGA4 about LSCC.

carbon metabolism in cancer, and DNA replication, indicating the mechanisms of ZJC in the treatment of LSCC. The other signaling pathways included prostate cancer, protein processing in endoplasmic reticulum, spliceosome, transcriptional misregulation in cancer, and ubiquitin-mediated proteolysis (Figure 5). Through the MCODE plugin, two main modules of the core PPI network were obtained, one of which was functionally enriched in alcoholism, transcriptional misregulation in cancer, and systemic lupus erythematosus (Figure 6).

3.7. LSCC Survival Analysis. To demonstrate the relationship between key genes and LSCC, we analyzed the genes in core module through the GEPIA online database and Kaplan-Meier curve. We found that LSCC patients with high expression of HIST1H3J, HIST1H3F, and ITGA4 had worse overall survival, while LSCC patients with high expression of NTRK1, COPS5, HIST1H3A, and HIST1H3G had significantly worse disease-free survival (Figure 7).

\section{Discussion}

Based on the network pharmacology analysis of drug and disease target, collateral relationship can effectively reveal the mechanism of ZJC in the treatment of LSCC. Here, we found 96 candidate targets of ZJC and 81 DEGs of LSCC. Then, we constructed the PPI network for them separately. The huge genes involved in the interacted PPI network were analyzed to derive the possible mechanisms of anti-LSCC of ZJC, including transcriptional misregulation cancer, alcoholism, and cell cycle.

In our study, we identified 11 active ingredients of ZJC, which synergistically regulated 96 candidate targets. A large number of published literatures showed that the 11 active ingredients had anticancer activities, respectively. As reported, fisetin could inhibit the proliferation and migration of human laryngeal cancer via ERK1/2 and AKT/NF-KB/mTOR signaling pathways and induce apoptosis in human lung cancer through the MAPK signaling pathway $[15,16]$. It was also revealed that kaempferol and quercetin were potential to inhibit cell migration and invasion in human head and neck 
squamous cell carcinoma $[17,18]$. Li et al. emphasized that taxifolin may arrest aggressive breast cancer by promoting the MET progress through decreasing the expression of $\beta$-catenin [19]. Additionally, the inhibitory potency of flavanone on human breast cancer and gastric cancer has been reported previously [20, 21]. To our knowledge, no previous studies have explored the synergistic effect of the 11 active ingredients deriving from ZJC in suppressing LSCC development.

To investigate the possible mechanism of anti-LSCC of ZJC at a system level, we applied GlueGO to complete KEGG enrichment signaling pathway analysis, through analyzing the huge targets of the core PPI network in tight corresponding to LSCC and ZJC. We identified 11 items, in particular, transcriptional misregulation in cancer, alcoholism, cell cycle, and central carbon metabolism in cancer (all $P<0.01$ ). It is apparent that both signal pathways of transcriptional misregulation in cancer and central carbon metabolism in cancer were closely associated with cancer $[22,23]$. Sequentially, transcriptional misregulation in cancer was the most significant pathway following ZJC acting on LSCC $(P<0.001)$. As reported, cancer is more likely to occur in the mucous membrane in direct contact with alcohol; therefore, an intermediate increase in the risk of laryngeal cancer was found among alcoholics [24]. Aberrant cell cycle results in uncontrolled proliferation of cells, which is the common nature of cancer [25]. Zhou et al. demonstrated that Erchen decoction plus Huiyanzhuyu decoction was promising medicine in treatment of LSCC through inhibiting the cell cycle and inducing apoptosis of LSCC cells [26]. Protein processing in endoplasmic reticulum (ER) is crucial for the pathogenesis of cancer, with severe ER stress closely related to the development and invasion of cancer $[27,28]$. These findings were consistent with the network pharmacology analysis.

\section{Conclusion}

Our study revealed that the anti-LSCC mechanism of ZJC was closely connected to transcriptional misregulation cancer, alcoholism, and cell cycle signaling pathway, which provided an important basis for further discussion of the follow-up experiment al design, making the experimental research more reasonable and more instructive.

\section{Data Availability}

The original data series GSE84957 used to support the findings of this study is downloaded from the Gene Expression Omnibus (GEO) microarray dataset.

\section{Conflicts of Interest}

The authors declare that they have no conflicts of interest.

\section{Authors' Contributions}

Feng Xiang conceived the study and drafted the manuscript. Guiyuan Peng analyzed and interpreted the data and reviewed the manuscript. Shasha Li instructed the research and reviewed the manuscript. Linman $\mathrm{Li}$ and Jieling Lin contributed to conception and design of the study. All authors revised, read, and approved the manuscript.

\section{References}

[1] H. R. J. Pukander and J. Pukander, "Symptoms of laryngeal carcinoma and their prognostic significance," Acta Oncologica, vol. 39, no. 2, pp. 213-216, 2009.

[2] L. D. R. Thompson and D. R. Lester, "Laryngeal dysplasia, squamous cell carcinoma, and variants," Surgical Pathology Clinics, vol. 10, no. 1, pp. 15-33, 2017.

[3] K. D. Hunter, E. K. Parkinson, and P. R. Harrison, "Profiling early head and neck cancer," Nature Reviews Cancer, vol. 5, no. 2, pp. 127-135, 2005.

[4] J. Zhu, S. Fedewa, and A. Y. Chen, "The impact of comorbidity on treatment (chemoradiation and laryngectomy) of advanced, nondistant metastatic laryngeal cancer," Archives of Otolaryngology-Head \& Neck Surgery, vol. 138, no. 12, pp. 1120-1128, 2012.

[5] J.-P. Zhang, X.-H. Tian, Y.-X. Yang et al., "Gleditsia species: an ethnomedical, phytochemical and pharmacological review," Journal of Ethnopharmacology, vol. 178, no. 3, pp. 155-171, 2016.

[6] S. J. Moon, Y. H. Cho, H. Kim et al., "Inhibitory effects of the ethanol extract of Gleditsia sinensis thorns on human colon cancer HCT116 cells in vitro and in vivo," Oncology Reports, vol. 22, no. 06, 2009.

[7] L. M. C. Chow, J. C. O. Tang, I. T. N. Teo et al., "Antiproliferative activity of the extract of Gleditsia sinensis fruit on human solid tumour cell lines," Chemotherapy, vol. 48, no. 6 , pp. 303-308, 2002.

[8] K. C. Tang, K. Y. Lam, S. Law et al., "The inhibitory effect of Gleditsia sinensis on cyclooxygenase-2 expression in human esophageal squamous cell carcinoma," International Journal of Molecular Medicine, vol. 23, no. 1, pp. 121-129, 1998.

[9] X. Liang, H. Li, and S. Li, "A novel network pharmacology approach to analyse traditional herbal formulae: the Liu-WeiDi-Huang pill as a case study," Molecular BioSystems, vol. 10, no. 5, pp. 1014-1022, 2014.

[10] N. Wang, Y. Zheng, J. Gu et al., "Network-pharmacologybased validation of TAMS/CXCL-1 as key mediator of XIAOPI formula preventing breast cancer development and metastasis," Scientific Reports, vol. 7, no. 1, p. 14513, 2017.

[11] A. Y. Lee, W. Park, T.-W. Kang, M. H. Cha, and J. M. Chun, "Network pharmacology-based prediction of active compounds and molecular targets in Yijin-tang acting on hyperlipidaemia and atherosclerosis," Journal of Ethnopharmacology, vol. 221, no. 15, pp. 151-159, 2018.

[12] X. Liu, S. Ouyang, B. Yu et al., "PharmMapper server: a web server for potential drug target identification using pharmacophore mapping approach," Nucleic Acids Research, vol. 38, no. 2, pp. W609-W614, 2010.

[13] A. Martin, M. E. Ochagavia, L. C. Rabasa, J. Miranda, J. Fernandez-de-Cossio, and R. Bringas, "BisoGenet: a new tool for gene network building, visualization and analysis," Bmc Bioinformatics, vol. 11, no. 1, pp. 91-99, 2010.

[14] Z. Tang, C. Li, B. Kang, G. Gao, C. Li, and Z. Zhang, "GEPIA: a web server for cancer and normal gene expression profiling and interactive analyses," Nucleic Acids Research, vol. 45, no. W1, pp. W98-W102, 2017.

[15] X.-J. Zhang and S.-S. Jia, "Fisetin inhibits laryngeal carcinoma through regulation of $\mathrm{AKT} / \mathrm{NF}-\kappa \mathrm{B} / \mathrm{mTOR}$ and ERK1/2 signaling pathways," Biomedicine \& Pharmacotherapy, vol. 83, pp. 1164-1174, 2016. 
[16] A. Klimaszewska-Wisniewska, M. Halas-Wisniewska, T. Tadrowski, M. Gagat, D. Grzanka, and A. Grzanka, "Paclitaxel and the dietary flavonoid fisetin: a synergistic combination that induces mitotic catastrophe and autophagic cell death in A549 non-small cell lung cancer cells," Cancer Cell International, vol. 16, no. 1, 2016.

[17] C.-Y. Chan, C.-H. Lien, M.-F. Lee, and C.-Y. Huang, "Quercetin suppresses cellular migration and invasion in human head and neck squamous cell carcinoma (HNSCC)," Biomedicine, vol. 6, no. 3, p. 15, 2016.

[18] H. I. Swanson, E.-Y. Choi, W. B. Helton, C. G. Gairola, and J. Valentino, "Impact of apigenin and kaempferol on human head and neck squamous cell carcinoma," Oral Surgery, Oral Medicine, Oral Pathology and Oral Radiology, vol. 117, no. 2, pp. 214-220, 2014.

[19] J. Li, L. Hu, T. Zhou et al., "Taxifolin inhibits breast cancer cells proliferation, migration and invasion by promoting mesenchymal to epithelial transition via $\beta$-catenin signaling," Life Sciences, vol. 232, p. 116617, 2019.

[20] C. H. Park, E. R. Hahm, J. H. Lee, K. C. Jung, and C. H. Yang, "Inhibition of $\beta$-catenin-mediated transactivation by flavanone in AGS gastric cancer cells," Biochemical and Biophysical Research Communications, vol. 331, no. 4, pp. 1222-1228, 2005.

[21] E. Kim, J. Lee, and G. Kim, "Anti-carcinogenic effect of a new analogue 4'-chloroflavanone from flavanone in human breast cancer cells," International Journal of Molecular Medicine, vol. 25, no. 2, pp. 293-298, 2009.

[22] T. I. Lee and R. A. Young, "Transcriptional regulation and its misregulation in disease," Cell, vol. 152, no. 6, pp. 1237-1251, 2013.

[23] T. L. Wong, N. Che, and S. Ma, "Reprogramming of central carbon metabolism in cancer stem cells," Biochimica Et Biophysica Acta Molecular Basis of Disease, vol. 1863, no. 7, pp. $1728-1738,2017$.

[24] A. J. McMichael, "Increases in laryngeal cancer in Britain and Australia in relation to alcohol and tobacco consumption trends," Lancet, vol. 311, no. 8076, pp. 1244-1247, 1978.

[25] T. Otto and S. Piotr, "Cell cycle proteins as promising targets in cancer therapy," Nature Reviews Cancer, vol. 17, no. 2, pp. 93-115, 2017.

[26] S. Q. Zhou, Q. L. Luo, X. Tan et al., "Erchen decoction plus huiyanzhuyu decoction inhibits the cell cycle, migration and invasion and induces the apoptosis of laryngeal squamous cell carcinoma cells," Journal of Ethnopharmacology, vol. 256, 2020.

[27] H. W. Moon, H. G. Han, and Y. J. Jeon, "Protein quality control in the endoplasmic reticulum and cancer," International Journal of Molecular Sciences, vol. 19, no. 10, p. 3020, 2018.

[28] E. Mohamed, Y. Cao, and P. C. Rodriguez, "Endoplasmic reticulum stress regulates tumor growth and anti-tumor immunity: a promising opportunity for cancer immunotherapy," Cancer Immunology Immunotherapy Cii, vol. 66, pp. 1069-1078, 2017. 